\title{
Hallazgos Histológicos en Hemorragia Uterina Disfuncional
}

\author{
Drs.: Carlos Cortes Caballero M.D.*, Ruben Serrano Gómez M.D.** \\ Alvaro Gamboa $\mathrm{G}^{* * *}$
}

\section{Introducción}

El cuadro descrito por Schoeder (1) llamado Metropatía Hemorrágica tiene características especiales como: "persistencia folicular, falta de ovulación, hiperplasia glandular quística del endometrio y descamación"; por eso hemos preferido el nombre de Hemorragia Uterina Disfuncional, por lo descriptivo desde el punto de vista clínico, ya que en realidad no investigamos los cambios concomitantes que se suceden a nivel del ovario. Sornos conscientes por ello de la limitación de nuestras observaciones y nos apartamos del concepto expresado por Botella (2) de que el "diagnóstico definitivo en estos casos lo arroja el estudio de endometrio". Sabemos que estos hallazgos son solo la manifestación de una anormalidad o disfunción endocrina de fondo, como ya lo había enfatizado Novak (3), que compromete el eje hipotálamo, hipófisis y ovario (4).

\footnotetext{
* Patólogo ISS - Bucaramanga

* Ginecobstetra ISS - Bucaramanga, Ginecólogo Caja Nal. Bucaramanga.

** Profesor Asociado Facultad de Salud UIS - Bucaramanga.
}

\section{Métodos}

Se revisaron 100 historias clínicas de mujeres que fueron sometidas a curetaje por "sangrado anormal", es decir, fuera del período menstrual, sin tener en cuenta antecedentes familiares, personales o ginecoobstétricos; tampoco hubo limitación en cuanto a procedencia, edad - raza. La mayoría de ellas son usuarias del. ISS; el procedimiento quirúrgico fue practicado por distintos especialistas y analizado por el mismo patólogo, quién sin conocimientos de la historia clínica los clasificó en:

a) Endometrios normales. Estrogénicos o Progestacionales.

b) Endometrios hiperestrogénicos o Persistentes.

c) Endometrios hiperplásicos.

Las hiperplasias fueron agrupadas de acuerdo a Gusberg (5) en grados I - II -III-IV - según la atipia encontrada.

Se aplicó la fórmula de CHI-CUADRADO (6) con el fin de analizar los cuadros y averiguar si estadísticamente existe o no relación entre las diferentes variables estudiadas. 


\section{Discusión}

Tabla No. 1

\section{EDAD SEGUN IRREGULARIDAD DEL CICLO}

\begin{tabular}{|c|l|l|c|c|}
\hline Edad & $R$ & $I$ & Total & $\%$ Irregularidad \\
\hline $20-24$ & 6 & 7 & 13 & 53.8 \\
\hline $25-29$ & 20 & 14 & 34 & 41.2 \\
\hline $30-34$ & 6 & 6 & 12 & 50.0 \\
\hline $35-39$ & 3 & 11 & 14 & 78.5 \\
\hline $40-44$ & 7 & 7 & 14 & 50.0 \\
\hline $45-49$ & 1 & 6 & 7 & 85.7 \\
\hline $50-54$ & 2 & 4 & 6 & 66.7 \\
\hline Total & 45 & 55 & 100 & 55.0 \\
\hline
\end{tabular}

R: Regularidad I: Irregularidad $X^{2}$ Cal. $=11.8397$

$$
x^{2}, 5 \%, 6=12.59
$$

En el grupo se halló que el $55 \%$ presentó ciclo irregular.

Dentro de la población estudiada se observa tendencia al aumento en el porcentaje de la irregularidad en el ciclo a medida que pasan los años. Las mujeres de edad extrema superior tienen proporción de irregularidad de más de un $70 \%$ promedio.

A pesar de existir diferencias algo apreciables en la irregularidad el valor obtenido de $X^{2}$ nos dice que no existen diferencias significativas y que las variaciones de dichos porcentajes no son reales.

Llama la atención que la mayoría de nuestras pacientes están en la edad reproductiva $(83 \%)$, lo cual contrasta con otras experiencias donde el sangrado se sucede en un $50 \%$ de los casos por encima de los 45 años (7).

Es importante recalcar que ninguna de las muestras fue insuficiente para el diagnóstico y que no se encontró pato- logía endometrial asociada. Sin tener en cuenta el ciclo podemos decir que la mayoría de las muestras fueron morfológicamente anormales $(52 \%)$. En otros estudios de material similar predominan los endometrios normales $(63 \%)(8)$.

Se observa más alta frecuencia de la anormalidad del ciclo cuando hay un endometrio con alteraciones en el nivel de estrógeno, el cual es más acentuado en los casos de hiperplasia. Este hallazgo concuerda con otros (9). Del total de mujeres con variaciones en el ciclo hay 30 (54.5) que presentan cambios endometriales que se apartan de la normalidad (E. persistente e hiperplasia) en cambio este porcentaje es menor $(40 \%)$ cuando el ciclo es regular.

Al igual que el caso anterior encontramos que hay diferencias significativas cuando aplicamos chi-cuadrado.

Tabla No. 2

\section{CAMBIOS ENDOMETRIALES SEGUIN IRREGULARIDAD DEL CICLO}

\begin{tabular}{|l|r|r|l|c|}
\hline & R & I & Total & $\%$ I / T \\
\hline 1. Progestacional & 6 & 7 & 16 & 43.8 \\
\hline 2. Estrogénico & 16 & 16 & 32 & 50.0 \\
\hline 3. E. persistente & 11 & 17 & 28 & 60.1 \\
\hline 4. Hiperplasia & 7 & 13 & 20 & 65.0 \\
\hline 5. Otros & 2 & 2 & 4 & \\
\hline Total & 45 & 55 & 100 & 55.0 \\
\hline
\end{tabular}

$$
X^{2} \text { Cal. }=3.37 \quad X^{2}, 5 \%, 3=7.81
$$

La mayoría de las madres escogidas tienen menos de 3 hijos $(50 \%)$.

La anormalidad del ciclo se encuentra altamente asociada con las mujeres de una paridad media entre $3-5$ hijos, esto lo refuerza la prueba estadística, la cual 
nos dice que sí hay diferencias significativas, o sea que si hay alguna relación entre la multiparidad e irregularidad.

Aunque las mujeres con mayor multiparidad tienen una incidencia menor $(50 \%)$ que las promedio, ella se conserva más alta que en las madres con escasos hijos.

Tabla No. 3

MULTIPARIDAD SEGUN IRREGULARIDAD DEL CICLO

\begin{tabular}{|rr|r|c|c|}
\hline & $\mathrm{R}$ & $\mathrm{I}$ & $\mathrm{T}$ & $\%$ de Irregularidad \\
\hline $0-2$ & 27 & 22 & 49 & 42.9 \\
\hline $3-5$ & 9 & 24 & 33 & 75.8 \\
\hline $6-7$ & 9 & 9 & 18 & 50.0 \\
\hline & 45 & 55 & 100 & 55.0 \\
\hline
\end{tabular}

$$
X^{2} \text { Cal. }=6.41 \quad X^{2} 5 \%, 2=5.99
$$

La manifestación clínica más frecuente es la hipermenorrea (41 casos), la menos frecuente la polimenorrea (8 casos).

Por otra parte observamos que la irregularidad del ciclo no está asociada aparentemente a algún tipo de trastorno, siendo esos porcentajes casi invariables aunque hay una ligera elevación en la irregularidad cuando hay polimenorrea sin embargo, estadísticamente no hay diferencias significativas.

Sobresale ra clara tendencia de aumentar el riesgo de hiperplasia a medida que envejece la mujer; pasamos de mujeres jóvenes con un $14.9 \%$ de hiperplasia a un $50 \%$ en mujeres viejas.

La docimasia estadística nos muestra que no hay relación entre la edad $y$ cambios endometriales y por consiguiente las variables se consideran independientes.

Tabla No. 4

\section{TRASTORNOS SEGUN EL CICLO}

\begin{tabular}{|l|r|r|r|r|}
\hline $\begin{array}{l}\text { Trastornos } \\
\text { Hemorrágicos }\end{array}$ & $\mathbf{R}$ & $\mathbf{I}$ & $\mathbf{T}$ & $\%$ I / T \\
\hline $\mathrm{H}$ & 20 & 20 & 41 & 48.8 \\
\hline $\mathrm{P}$ & 3 & 5 & 8 & 62.5 \\
\hline $\mathrm{M}$ & 12 & 12 & 24 & 50.0 \\
\hline Mixta & 9 & 18 & 27 & 66.7 \\
\hline Total & 45 & 55 & 100 & 55.0 \\
\hline
\end{tabular}

$\mathrm{X}^{2}$ Cal. $=0.27$

$X^{2}, 5 \%, 3=7.81$

$\mathrm{H}_{1}$ Hipermenorrea

P: Polimenorrea

M: Metrorragia

Tampoco encontramos alguna relación entre los cambios endometriales y el número de hijos habidos en la madre. Estadísticamente los valores de $\mathrm{CHI}$ CUADRADO insinúan independencia entre las variables analizadas; en consecuencia ellos no están asociados.

Tabla No. 5

\section{CAMBIOS ENDOMETRIALES SEGUN LA EDAD}

\begin{tabular}{|l|c|c|c|c|c|c|}
\hline Gestación & Prog. & Est. & E.P. & H & Otros & Total \\
\hline $0-2$ & 8 & 20 & 13 & 4 & 2 & 47 \\
\hline $3-5$ & 3 & 9 & 11 & 11 & 1 & 35 \\
\hline $6-+$ & 5 & 3 & 4 & 5 & 1 & 18 \\
\hline Total & 16 & 32 & 28 & 20 & 4 & 100 \\
\hline
\end{tabular}

$\mathrm{X}^{2} \mathrm{Cal} .=11.09 \quad \mathrm{X}^{2}, 5 \%, 9=16.92$ 
Tabla No. 6

\section{CAMBIOS ENDOMETRIALES SEGUN LA GESTACION}

\begin{tabular}{|l|c|r|r|r|c|c|}
\hline Edad & Prog. & E & EP & H & Otros & Total \\
\hline $20-29$ & 6 & 16 & 15 & 7 & 3 & 47 \\
\hline $30-39$ & 3 & 10 & 8 & 5 & 0 & 26 \\
\hline $40-49$ & 6 & 5 & 5 & 5 & 0 & 21 \\
\hline $50-58$ & 1 & 1 & - & 3 & 1 & 6 \\
\hline Total & 16 & 32 & 28 & 20 & 4 & 100 \\
\hline
\end{tabular}

$X^{2}$ Cal. $=8.52 \quad X^{2}, 5 \%, 6=12,59$

\section{Resumen}

Se revisaron las historias cl ínicas y los hallazgos histólogicos de 100 mujeres con hemorragia uterina disfuncional.
El $55 \%$ de ellas presentó ciclo irregular, hallazgos más frecuentes a medida que pasan los años. La mayoría de los endometrios fueron anormales $(52 \%)$. A mayor paridad más irregularidad en el ciclo. La manifestación clínica más frecuente fue la hipermenorrea $(41 \%)$ ); la menos frecuente la polimenorrea. La hiperplasia está relacionada con la edad.

\section{HISTOLOGICAL. FINDINGS IN DYSFUNCTIONAL UTERINE BLEEDING}

\section{Summary}

The authors analyzed the medical records and histological findings of 100 women with dysfunctional uterine bleeding.

$55 \%$ of the women presented irregular menstrual cycles, frequently more so as age increased. Most endometria were abnormal $(52 \%)$.

The cycles were more frequently irregular when parity was higher. $\mathrm{Hy}$ permenorrhea was the clinical symptom most often found $(41 \%)$, and polymenorrhea was the least frequent.

Hyperplasia is clearly related to age.

\section{Bibliografía}

1. SCHOEDER, R. Arch Gynak 104, 27 (1915).

2. BOTELLA LLUSIA JOSE. Endocrinología de la Mujer pág. 512, Editorial

Científico Médica. Barcelona. Cuarta edición 1966.

3. NOVAK and WOODRUFF. Gynecology and Obstetric Pathology. 
4. HENDRICKSON R.M., Kempson R. L., Surginal Pathology of the uterine corpus W.B. Saunders, Philadelphia, London, Toronto 1980 pg. 250.

5. GUSBERG SAUL B. M.D. The individual at high risk for endometrial Carcinoma. Am. J. Obstet. Gynecol 125: 535 November 1976.

6. YA LUM CHOU. Análisis Estadístico México 1972. 1a. Edición pg. 497.
7. BOTERO U.J., JUBIZ H.A., HENAO G.F. Ginecología y Obstetricia Texto Integrado Tomo I. segunda edición 1980 pg.6.

8. HORTA PARRAGUEZ SAUL. La aspiración endouterina como procedimiento diagnóstico y terapéutico en Ginecología y Obstetricia. Tendencias actuales en la regulación de la fertilidad. Total Graphics, Inc. 1977 pág. 45-49. 